\title{
Analysis of general surgery outpatient clinic admissions and operations during COVID-19 pandemic in Turkey: Reactions of 12728 patients
}

\author{
Ferahman S, Dural AC, Aydin H, Sahbaz NA, Akarsu C, Peker KD, Donmez T, Karabulut M \\ Department of General Surgery, Ministry of Health Bakirkoy Dr Sadi Konuk Training \\ and Research Hospital, Istanbul, Turkey. sinaferahmantr@hotmail.com
}

\begin{abstract}
Covid-19 pandemic is spreading rapidly in Turkey. We aimed to examine the numbers and demographic data of patients who applied to the general surgery outpatient clinics and operated in this process. Our primary outcome is to reveal the response of general surgery patients to the Covid-19 pandemic.

The first Covid-19 case in Turkey has appeared in March 11, 2020. Patients who were operated on due to a surgical emergency or trauma were evaluated separately. Patients in 3 periods were compared with each other. A total of 12728 patients were examined in general surgery outpatient clinics in 26 working days. It is seen that patients come to the outpatient clinic after the first time the Covid-19 patient is seen. All patients reduced hospitalization after the first death due to Covid-19. Women had reduced going to the hospital earlier than men. There was no change in the number of emergency surgeries. Rapid decrease was observed in the number of elective surgeries.

It is not easy to control the entrance and exit of these busy hospitals. The remote diagnosis (mail, phone or video-call) and treatment methods that can be expected in the near future may be even closer with the Corona virus (Tab. 3, Fig. 4, Ref. 15). Text in PDF www.elis.sk KEY WORDS: general surgery, Covid-19, outpatient clinics, operation, reaction of patients.
\end{abstract}

\section{Introduction}

The Covid-19 virus infection which emerged from Wuhan, China, causing pandemics has changed the working habits of health centers all around the world. Hospitals began to treat increasing numbers of Covid-19 patients by delaying elective procedures as much as possible. As pulmonary diseases, infectious diseases departments and intensive care units were insufficient for patients with Covid-19, other departments rearranged their schedule, and their patient admissions were minimized and beds were provided for patients diagnosed with Covid-19. In addition, patients who applied to the intensive care unit for other reasons were stabilized and discharged as quickly as possible $(1,2)$.

In order to take precautions regarding coronavirus, a committee was established on January 10, 2020, under the direction of the Ministry of Health, with 31 medical scientists in specialties such as infectious diseases, microbiology, virology, internal diseases, intensive care and pulmonary diseases. The decisions taken by this board were put into practice day by day. In this context, after the diagnosis of the first case, schools were vacationed, bars, restaurants and entertainment venues were closed, and sports competi-

Department of General Surgery, Ministry of Health Bakirkoy Dr Sadi Konuk Training and Research Hospital, Istanbul, Turkey

Address for correspondence: S. Ferahman, Bakirkoy Dr. Sadi Konuk Egitim ve Arastirma Hastanesi, Genel Cerrahi 4. Kat Zuhuratbaba Mah. Tevfik Saglam Cd. No:11 Bakirkoy, Istanbul, Turkey.

Phone: +90.533 .6120201$ tions were canceled. In order to prevent contamination, people were warned to stay at home trough written, visual and social media.

A week after the first case, The Ministry of Health has issued a notification to stop elective procedures in all health care centers and extend the routine follow-up of chronic patients as much as possible and direct them to family physicians. Warnings have also been made for people not to apply to the hospitals unless it is mandatory, in order to reduce the patient load of hospitals. Reducing hospital admissions was important for two reasons; the first one was to reduce the workload of healthcare workers, and the second one was to prevent hospital-based spread. Because of the rapid spread of Covid-19, hospitals have become an important source of transmission (3).

General surgery department of our hospital has 4 gastroenterology \& obesity (GIO), 2 breast surgery, 1 endocrine surgery and 1 hepatobiliary surgery outpatient clinic serving during the daytime hours. In the evening, patients are cared for in 2 outpatient clinics between 5 p.m. and 8 p.m. A total of 10 outpatient clinics provide service during the day and the evening.

Three operating rooms are actively working on working days. In addition, emergency and trauma surgeries are performed in 1 emergency room every day. An average of 250 elective general surgery and 150 emergency operations are performed in a month. Our hospital is a 3rd level state hospital and a level 1 trauma center that serves as a training hospital (4).

With the spread of the Covid-19 infections all over the country, our hospital has become one of the most important centers for 


\section{5-480}

those patients. On March 11, 2020, first Covid-19 case was diagnosed in our country and on March 18, the first Covid-19 patient was deceased.

Istanbul is a large metropolis with a population of 15 million. An average of 15 million tourists visit this crowded city annually. There are 159 private, 15 university hospitals and 371 hospitals affiliated to the Ministry of Health. Our hospital is an easily accessible hospital in the center of Istanbul. Our general surgery department has the highest number of outpatient admissions in Istanbul, with more than 10,000 applications per month.

In this study, we aimed to examine the numbers and demographic data of patients who applied to the general surgery outpatient clinics and operated in this process. Our primary outcome is to reveal the response of general surgery patients to the Covid-19 pandemic.

\section{Methods}

The data of patients aged 18 and over who applied to the general surgery clinic of our hospital between 21.02.2020 and 27.03.2020 were reviewed retrospectively. Patients admitted to the general surgery outpatient clinics and the demographic data of these patients were evaluated 13 days before and 13 days after the first Covid-19 case. Since the elective patient clinic was not held on weekends, a total of 14 days
Tab. 1. Demographics and distribution of outpatient clinic admissions, surgical operations and indications of procedures in our department during COVID-19 spread in Turkey.

\begin{tabular}{|c|c|c|c|c|}
\hline Parameter & $\begin{array}{l}\text { Period } 1^{*} \\
(\mathrm{n}=8599)\end{array}$ & $\begin{array}{l}\text { Period 2** } \\
(\mathrm{n}=2612)\end{array}$ & $\begin{array}{l}\text { Period } 3^{\dagger} \\
(\mathrm{n}=1517)\end{array}$ & $\begin{array}{c}\text { TOTAL } \\
(\mathrm{n}=12728)\end{array}$ \\
\hline Length of Period (Days) & 13 days & 5 days & 8 days & 26 days \\
\hline $\begin{array}{l}\text { Number of Daily Patient } \\
\text { Admissions (n, Mean } \pm \text { SD) }\end{array}$ & $661.4 \pm 78.9$ & $522.4 \pm 88.6$ & $189.6 \pm 106.8$ & $489.5 \pm 103.2$ \\
\hline \multicolumn{5}{|l|}{ Gender (n) } \\
\hline Female & 6361 & 1916 & 989 & 9266 \\
\hline Male & 2231 & 700 & 531 & 3462 \\
\hline Age (years, Mean \pm SD) & $51.1 \pm 16.1$ & $41.4 \pm 13.1$ & $46.6 \pm 15.6$ & $49.0 \pm 16.0$ \\
\hline \multicolumn{5}{|l|}{ Age category $(\mathrm{n} / \%)$} \\
\hline $18-30$ & $906 / 10.5$ & $281 / 10.8$ & 173/11.4 & 1356 \\
\hline $30-40$ & $1311 / 15.3$ & $432 / 16.5$ & $255 / 16.8$ & 1997 \\
\hline $40-50$ & $2058 / 23.9$ & $637 / 24.4$ & $397 / 26.2$ & 3095 \\
\hline $50-60$ & $1825 / 21.2$ & $534 / 20.4$ & $314 / 20.7$ & 2677 \\
\hline $60-70$ & $1463 / 17.0$ & $438 / 16.8$ & $237 / 15.6$ & 2134 \\
\hline $70-80$ & $755 / 8.8$ & $219 / 8.4$ & $103 / 6.8$ & 1080 \\
\hline $80+$ & $281 / 3.3$ & $71 / 2.7$ & $38 / 2.5$ & 389 \\
\hline \multicolumn{5}{|l|}{ Outpatient Clinic Admissions (n/\%) } \\
\hline Breast & $2423 / 28.18$ & $779 / 29.82$ & $433 / 28.54$ & $3635 / 28.6$ \\
\hline Endocrine & $1104 / 12.84$ & $291 / 11.14$ & $94 / 6.20$ & $1489 / 11.7$ \\
\hline Hepatobiliary & $1894 / 22.03$ & $585 / 22.40$ & $386 / 25.44$ & $2865 / 22.5$ \\
\hline $\mathrm{GIO}^{\ddagger}$ & $3178 / 36.96$ & $957 / 36.64$ & $604 / 39.82$ & $4739 / 37.2$ \\
\hline \multicolumn{5}{|l|}{$\begin{array}{l}\text { Surgical Operations by field (n/\%) } \\
\text { (n) }\end{array}$} \\
\hline Emergency & 77 & 26 & 30 & 133 \\
\hline Breast & $33 / 17,5$ & $5 / 10$ & $5 / 25$ & $43 / 16,6$ \\
\hline Endocrine & $32 / 16,9$ & $8 / 16$ & $3 / 15$ & $43 / 16,6$ \\
\hline Hepatobiliary & $45 / 23,8$ & $13 / 26$ & $2 / 10$ & $60 / 23,2$ \\
\hline GIO & $79 / 41,8$ & $24 / 48$ & $10 / 50$ & $113 / 43,6$ \\
\hline \multicolumn{5}{|l|}{$\begin{array}{l}\text { Elective/Emergengy Surgical } \\
\text { Operations }(\mathrm{n} / \%)\end{array}$} \\
\hline Elective & $189 / 71.1$ & $50 / 65.8$ & $20 / 40$ & $259 / 66.1$ \\
\hline Emergency & $77 / 28.9$ & $26 / 34.2$ & $30 / 60$ & $133 / 33.9$ \\
\hline \multicolumn{5}{|l|}{ Indications of Elective Procedures (n/\%) } \\
\hline Malignancy or Suspicious for malignancy & $67 / 35.4$ & $17 / 34$ & $18 / 90$ & $102 / 39.4$ \\
\hline Benign diseases & $122 / 64.6$ & $33 / 66$ & $2 / 10$ & $157 / 60.6$ \\
\hline
\end{tabular}

* Period 1: From February 21st, 2020 to March 11th, 2020 (when first COVID-19 case was diagnosed; 13 days)

** Period 2: From March 11th to March 18th, 2020 (when first COVID-19 case was deceased; 5 days)

$\uparrow$ Period 3: From March 18th to March 27th, 2020 (8 days)

\$ GIO: Including Upper GI, Colorectal and Obesity Surgery Outpatient Clinics

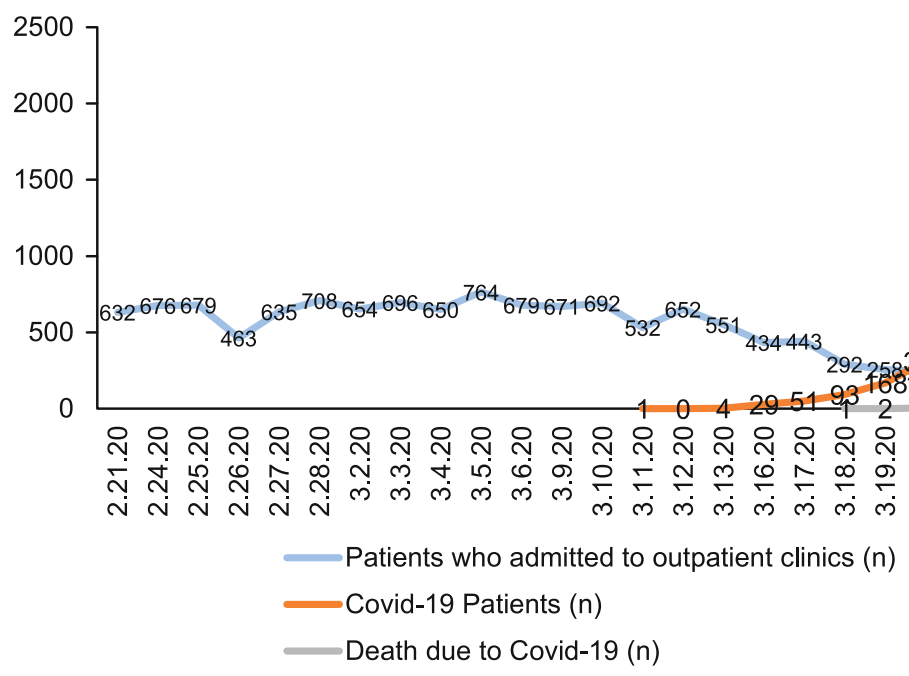

Fig. 1. The decreasing trend of daily patient admissions to general surgery outpatient clinics in our hospital during COVID-19 Pandemia and the first COVID-19 case was diagnosed at March 11th, 2020 and the first COVID-19 case was deceased at March 18th, 2020 in Turkey. 


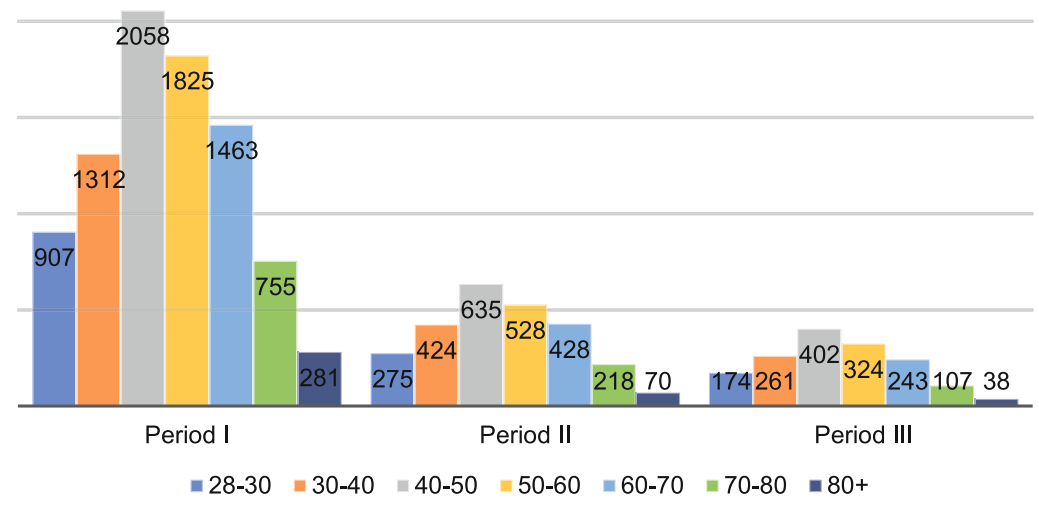

Fig. 2. The distribution and comparison of patients by age category who admitted to outpatient clinics of our department in three periods of COVID-19 spread.

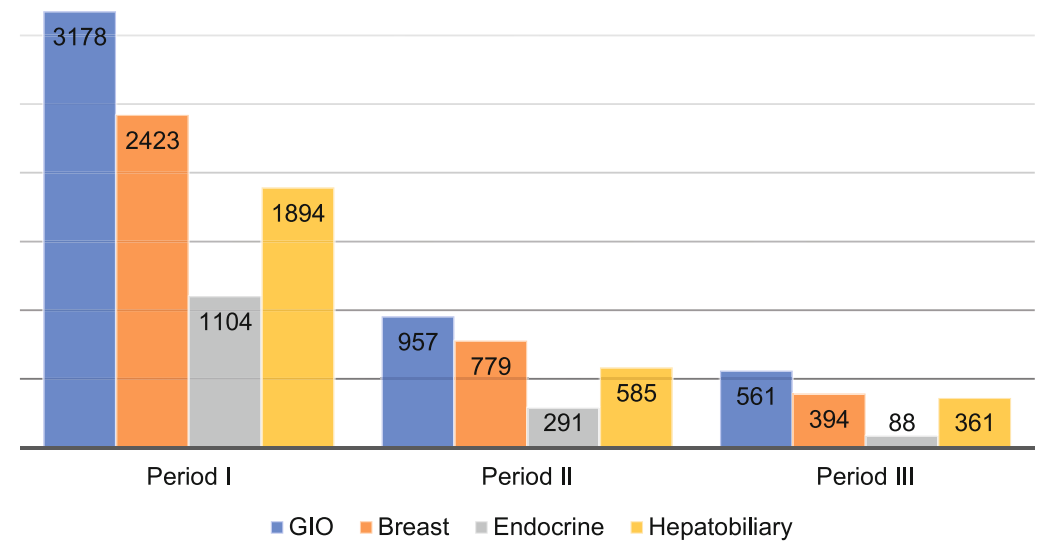

Fig. 3. The decreasing trend of outpatient clinic admissions by fields (Breast, Endocrine, Hepatobiliary and GIO* in three periods of COVID-19 Pandemia in Turkey. (*GIO is including Upper GI, Colorectal and Obesity Surgery Clinics).

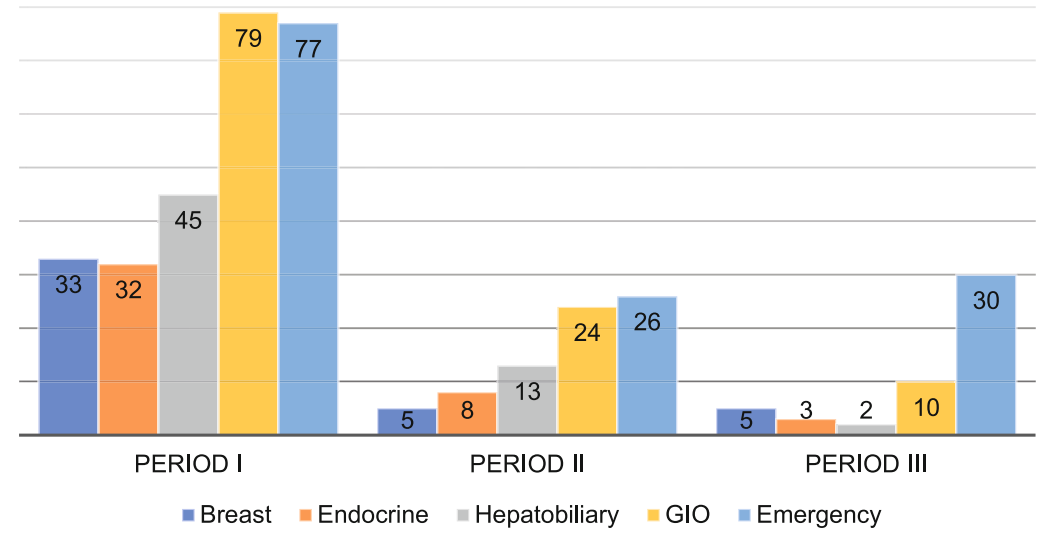

Fig. 4. The number of elective surgical procedures by field (Hepatobiliary, Endocrine, Breast and GIO*) and emergency procedures in our department during COVID-19 spread in Turkey. *GIO is including Upper GI, Colorectal and Obesity Surgery Clinics.
(Saturday and Sunday) were not included in the study (Fig. 1).

The first Covid-19 case in Turkey has appeared in March 11, 2020. We included 13 work-days before this date in the study, and called it Period I. Five days until March 18, 2020, the date of first Covid-19 related death, was termed as period II and 8 work days afterwards was called period III. Patients were grouped according to the gender and also according to their age as patients being between 18-30 years, 30-40 years, 40-50 years, 50-60 years, 60-70 years, 70-80 years, and above 80 years of age. Patients were assessed separately according to the outpatient clinics they applied to as breast, endocrine, hepatobiliary and GIO outpatient clinics (Tab. 1).

Information about elective and emergency surgeries performed in these periods was obtained from the electronic database of the hospital. Elective surgeries were divided according to the relevant surgical discipline (breast, endocrine, hepatobiliary and GIO). Preoperative diagnoses were grouped as malignant, benign and suspicious. Patients who were operated on due to a surgical emergency or trauma were evaluated separately. Patients in periods I, II and III were compared with each other.

All procedures performed in this study involving human participants were in accordance with the 1964 Helsinki declaration and its later amendments or comparable ethical standards. Informed consent was obtained from all individual participants included in the study. The authors also declare that they have no competing financial interests, and they do not have any conflict of interest.

Descriptive statistics was used to present the demographic characteristics of the study population. Differences between these groups were tested using Pearson chi-square test or Fisher's exact test for categorical variables, and one-way analysis of variance (ANOVA) for continuous variables. All analyses were performed using IBM SPSS Statistics version 24.0 (IBM Corp, Armonk, NY, USA). A p value $<0.05$ was considered statistically significant.

\section{Results}

A total of 12728 patients were examined in general surgery outpatient clinics 
in 26 working days. Female/male ratio was $9266 / 3462$. The average age of the patients was $49.37( \pm 15.99)$. When evaluated proportionally among age groups, patients in their 5th decade visited the outpatient clinics most in all periods (Fig. 2).

There are 2500 patients in the mean monthly hepatobiliary outpatient clinic, 3200 patients in the breast outpatient clinics, 1700 patients in the endocrine outpatient clinic and 2700 patients in the GIO outpatient clinics (Fig. 3).

Patient distribution among specific outpatient clinics are shown in Table 1. The average number of daily outpatient clinic applications was $661.4( \pm 78.99)$ patients before the first case of Covid-19 (period I), $522.4( \pm 88.58)$ patients between the first case and the first death (period II) and 189.6 $( \pm 106.79)$ patients after the first death (period III). When the number of applications to the general surgery outpatient clinics is evaluated, the most common application is to the general outpatient clinic, followed by breast, hepatobiliary and endocrine surgery outpatient clinics (Tab. 2). When patients who applied in period I, II and III were evaluated, there was no significant difference between periods I and II ( $p=$ 0.052 ). There was a significant decrease in applications between periods I and III $(p=0.001)$ and between the II and III $(p=0.001)$.

Application of women and men did not differ significantly between 1 st and 2 nd periods $(\mathrm{p}=0.073, \mathrm{p}=0.158)$, but there was a significant decrease between 1 st and 3 rd periods $(p=0.001, p$ $=0.001$ ). The number of patients also decreased in period I compared to the period III $(p=0.001)$. When women and men were compared with each other, women began to come to the outpatient clinic less than men $(p=0.001)$ (Tab. 3).

All age groups were compared with each other according to the number of applications. Admission to the outpatient clinics of all age groups has decreased. But there was no significant difference between age groups. When age groups were analyzed in subgroups according to gender, a significant decrease was noted in men aged 30-40 compared to women $(p=0.019)$. Between the ages of 70-80, the number of women coming to the outpatient clinic decreased significantly $(p=0.031)$. It was observed that men over 80 years of age came to the outpatient clinics less than women $(p=0.004)$.

The number of patients in breast, endocrine, hepatobiliary and GIO outpatient clinics decreased. A statistically significant decrease was observed in patients who applied to the endocrine clinic only between the first and second periods $(p=0.034)$ (Tab. $3)$. It was noteworthy that this decrease was the lowest in the hepatobiliary surgery while all patients were decreasing. The percentage of patients in the hepatobiliary polyclinic was $21.7 \%, 22.2$ $\%, 25.3 \%$, respectively, in periods I, II and III. In the analysis, there was a statistically significant increase in the percentage of
Tab. 3. Statistical comparison ${ }^{\S}$ of outpatient admission profiles and surgical procedures in our department by time periods of COVID-19 spread in Turkey.

\begin{tabular}{lccc}
\hline Parameter & $\begin{array}{c}\text { I vs II } \\
(p \text { value })\end{array}$ & $\begin{array}{c}\text { I vs III } \\
(p \text { value })\end{array}$ & $\begin{array}{c}\text { II vs III } \\
\text { ( } \text { value })\end{array}$ \\
\hline Mean number of Patients & 0.052 & $\mathbf{0 . 0 0 1}$ & 0.001 \\
\hline Gender & & & \\
$\quad$ Female & 0.073 & $\mathbf{0 . 0 0 1}$ & $\mathbf{0 . 0 0 1}$ \\
Male & 0.158 & $\mathbf{0 . 0 0 1}$ & $\mathbf{0 . 0 0 1}$ \\
\hline Age Category & & & \\
18-30 & 0.1 & $\mathbf{0 . 0 0 1}$ & $\mathbf{0 . 0 0 7}$ \\
30-40 & 0.229 & $\mathbf{0 . 0 0 1}$ & $\mathbf{0 . 0 0 1}$ \\
40-50 & 0.183 & $\mathbf{0 . 0 0 1}$ & $\mathbf{0 . 0 0 1}$ \\
50-60 & 0.129 & $\mathbf{0 . 0 0 1}$ & $\mathbf{0 . 0 0 2}$ \\
60-70 & 0.058 & $\mathbf{0 . 0 0 1}$ & $\mathbf{0 . 0 0 1}$ \\
70-80 & 0.142 & $\mathbf{0 . 0 0 1}$ & $\mathbf{0 . 0 0 1}$ \\
80+ & 0.314 & $\mathbf{0 . 0 0 1}$ & $\mathbf{0 . 0 1 1}$ \\
\hline Outpatient clinics by field & & & \\
Breast & 0.558 & $\mathbf{0 . 0 0 1}$ & $\mathbf{0 . 0 0 2}$ \\
Endocrine & 0.034 & 0.001 & 0.001 \\
Hepatobiliary & 0.256 & $\mathbf{0 . 0 0 1}$ & $\mathbf{0 . 0 0 1}$ \\
GIO* & 0.062 & $\mathbf{0 . 0 0 1}$ & $\mathbf{0 . 0 0 1}$ \\
\hline Surgical Procedures & & & \\
$\quad$ Emergency Surgery & 0.596 & 0.98 & 0.772 \\
Elective Surgery & 0.043 & 0.001 & 0.001 \\
Malignant/Benign & 0.968 & $\mathbf{0 . 0 0 1}$ & $\mathbf{0 . 0 0 1}$ \\
\hline
\end{tabular}

* GIO: Including Upper GI. Colorectal and Obesity Surgery Outpatient Clinics $\S$ Differences between these groups were compared using Pearson Chi-Square test or Fisher's Exact test for categorical variables and one-way analysis of variance (ANOVA) and Tukey's for continuous variables.

patients in the hepatobiliary outpatient clinics relative to the others $(p=0.010)$ (Fig. 3).

The patients who underwent surgical treatment were evaluated. The number of elective surgeries was 189 in period I, 50 in 
period II, and 20 in period III. The number of emergency surgeries is 70 in period I, 26 in period II and 30 in period III (Fig. 4). The ratio of malignant patients to all patients was $32 \%$ in the first period, $29 \%$ in the second period, and $88 \%$ in the third period (Tab. 1). When the number of elective surgeries was compared between the periods, it was observed that there was a statistically significant decrease $(p=0.043)$ between the first and second periods and a significant decrease $(p=0.001)$ between the second and third periods.

When malignant and benign patients were compared, there was no statistically significant difference between periods I-II $(p=0.968)$. It is observed that malignant patients between periods I-III and II-III underwent significantly more operations ( $\mathrm{p}=0.001$ and $\mathrm{p}=0.001)($ Tab. 3).

\section{Discussion}

The first patient in Turkey, has been working in the Grand Bazaar, one of Istanbul's top tourist destination. This patient and the people, who had been in close contact with him, were quarantined. It is important to find out, if the first patient was really the first patient? In publications, it has been shown that if we move backwards from patient zero, we can reach previous patients (5). These infected patients who have not been tested yet may have applied to outpatient clinics when their symptoms develop. In particular, hospitals are where virus transmission is experienced most intensely. Studies show that the hospital-acquired virus is 41 $\%$ during the SARS-COV-2 pandemic. For this reason, outpatient clinics of intensive hospitals are important places to transmit the disease (6).

Our study evaluating the number of people coming to the hospital shows that the patients continued to come to the hospital after the first patient was seen. During this time, the disease continued to spread. The public significantly reduced coming to the hospital only after the first death. We think, the reason for this is, that the patients can grasp the severity of the event only after the death is seen.

When we evaluate the patients in two groups as men and women, both of them continue coming to the outpatient clinics after the first Covid-19 case was seen, and only reduced after the first death. But women came to the hospital less compared to men. Especially in breast and endocrine outpatient clinics, female patient density is higher. We can attribute this to female patients coming to more annual routine checks. Patients without complaints did not prefer to come to the hospital in this process $(7,8)$.

All age groups were compared with each other according to the number of applications to the outpatient clinics. There was no significant difference between age groups. Age groups were examined in subgroups as men and women. A significant decrease was observed in men compared to women between the ages of 30-40 $(p=0.019)$. Between the ages of 70-80, the number of women coming to the outpatient clinic decreased significantly $(p=0.031)$. When patients are evaluated according to their ages, admission to the hospital decreased at all ages. Especially after March 22, 2020, a curfew was issued for people over 65 years. However, after this date, a proportionally significant difference was not observed in the patient group aged between $70-80$, and above 80 . Over the age of 80 , it was observed that men came to the outpatient clinics less than women ( $p=0.004)$ (Fig. 2). In studies, it is seen that older patients continue coming to the hospital $(9,10)$.

It is noteworthy that patients who applied to the endocrine outpatient clinic chose not to come to the hospital after the first case of Covid-19. However, there was an increase in the proportion of patients who applied to the hepatobiliary outpatient clinic (Fig. 3). Our hospital's hepatobiliary department is a reference center in Istanbul. In this process, patients continued to be directed from other health institutions.

We divided the operated patients during this period into two groups as emergency and elective. The number of elective surgeries, seen in Figure 4, decreased statistically during Period II ( $\mathrm{p}=$ $0.043)$ and it continued to decrease in Period III $(p=0.001)$. When elective surgeries are divided into benign and malignant, there is no significant difference between periods I and II $(\mathrm{p}=0.968)$, while there is a significant decrease between periods II and III $(\mathrm{p}=0.001)$. When the number of emergency operations was evaluated, no significant difference was found between the three periods (table 3 ). It would be suitable to explain the reason for the rapid decline in the number of elective surgeries, with the hospital management's decision to cease non-urgent operations after infection. As seen in the recent literature, this kind of management strategies were applied in many countries with a pandemic $(11,12)$.

Since the response of the public is slower than healthcare professionals, the number of patients coming to outpatient clinics has not decreased. They may continue to come as long as hospital services are not interrupted. Although the number of patients admitted to the outpatient clinic has minimally decreased, the number of emergency operations has not changed. The reason for this can be explained by the fact that our hospital is the one of the most important referral and trauma centers of the city.

Our hospital serves tens of thousands of patients daily, just like the central hospitals in other metropoles of the world. Due to its large capacity of treating patients with fully equipped and experienced healthcare professionals, it was chosen as the central hospital during the pandemic. Although infected and non-infected patients are physically separated within the hospital, patients still can be in contact with each other during transportation or in environmental areas. What we learned from the Covid-19 pandemic is that hospitals are quickly infected with this virus. Selected Pandemic hospitals' outpatient's clinics should be closed without waiting for the decisions of the patients. Elective surgeries can be stopped. Directing these patients to uninfected hospitals will reduce the speed of the disease's spread.

Reaching patients by post or telephone will reduce their arrival at the hospital. Although it is not possible to deny the importance of physical examination, tele-health practices applied in domestic branches in some countries may shed light on us. We should also consider applying to general surgery in the future, thereby reducing the number of hospital admissions. Perhaps routine controls after surgery will be done with blood samples and mobile radiography devices to be taken at home, and patients can be evalu- 


\section{5-480}

ated by video conferencing. The remote diagnosis and treatment methods that can be expected in the near future may be even closer with the Corona virus (10).

After a while, outpatient services and surgical interventions will stop in pandemic hospital. It is an option to stop the outpatient clinic and non-urgent surgical procedures early. Health institutions that are not particularly infected with the virus will be necessary for other patients $(13,14)$.

In conclusion it is not easy to control the entrance and exit of these busy hospitals. Considering that there are patients' relatives along with the patients, the number of people entering the hospital is much more than calculated. Controlled access to the hospital during a respiratory transmitted pandemic is an option to be evaluated. The body temperatures of the people (employees, patients and patient relatives) who come with a team to be established at the hospital entrances can be examined and the presence of symptoms can be questioned. Looking at the literature, this has been applied before and has been successful (15). Thus, suspicious patients can be separated earlier than other people and necessary precautions can be taken. With these precautions, we believe that the rate of spread can be slowed down. Entering the hospital should not be left to the preference of patients. Pandemic hospitals should be chosen by healthcare professionals and elective patients should be directed to uninfected hospitals.

\section{References}

1. Wang C, Horby PW, Hayden FG, Gao GF. A novel coronavirus outbreak of global health concern. The Lancet 2020; 395 (10223): 470-473.

2. Lenert L, Meswain BY. Balancing Health Privacy, Health Information Exchange and Research in the Context of the COVID-19 Pandemic. J Amer Med Inform Ass 2020.

3. Health MO. The current situation in Turkey. 2020; Available at: https:// covid19.saglik.gov.tr/\#.
4. The first 100-hospitals in each branch in connection with the outpatient clinic hospital and intensive care unit and emergency room data. 2017; Available at: https://dosyahastane.saglik.gov.tr/Eklenti/9300,2017-ocakekim-donemi-poliklinik-yatis-ve-yogun-bakim-ve-acil-servis-verileribaglaminda-her-bransta-ilk-100-hastanepdf.pdf?0.

5. Pépin J. The origins of AIDS: from patient zero to ground zero. 2013, BMJ Publishing Group Ltd.

6. Wang D, Hu B, Hu C et al. Clinical characteristics of 138 hospitalized patients with 2019 novel coronavirus-infected pneumonia in Wuhan, China. Jama, 2020.

7. Stavrakis AI, Ituarte PH, Ko CY, Yeh MW, Surgeon volume as a predictor of outcomes in inpatient and outpatient endocrine surgery. Surgery 2007; 142 (6): 887-899.

8. Kilickap S, Gumus M, Uncu D et al. Outcomes of Turkish National Breast Cancer Registry. 2017, American Society of Clinical Oncology.

9. Lin Y, Ji C, Weng W et al. Epidemiological and Clinical Characteristics of 124 Elderly Outpatients with COVID-19 in Wuhan, China. China (2/20/2020), 2020.

10. Sterpetti AV. Lessons Learned During the COVID-19 Virus Pandemic. Journal of the American College of Surgeons, 2020.

11. Iacobucci G. Covid-19: all non-urgent elective surgery is suspended for at least three months in England. 2020, British Medical Journal Publishing Group.

12. Cao Y, Li Q, Chen J et al. Hospital Emergency Management Plan During the COVID-19 Epidemic. Academic Emergency Medicine, 2020.

13. Tang $\mathbf{D}$, Tou $\mathbf{J}$, Wang $\mathbf{J}$ et al. Prevention and control strategies for emergency, limited-term, and elective operations in pediatric surgery during the epidemic period of COVID-19. World J Pediat Surg 2020; 3 (1): e000122.

14. Adalja AA, Toner E, Inglesby TV. Priorities for the US health community responding to COVID-19. Jama, 2020.

15. Xiao Y, Tan C, Duan J, Wu A, Li C. An Effective Model for the Outpatient Management of COVID-19. Infect Control Hosp Epidemiol 2020: 1-4. 